\title{
Polarized NIR observations of Sagittarius A*
}

\section{B. Shahzamanian*}

I.Physikalisches Institut, Universität zu Köln, Zülpicher Str.77, 50937 Köln, Germany Max-Planck-Institut für Radioastronomie, Auf dem Hügel 69, 53121 Bonn, Germany

E-mail: shahzaman@ph1.uni-koeln.de

\section{A.Eckart}

I.Physikalisches Institut, Universität zu Köln, Zülpicher Str.77, 50937 Köln, Germany Max-Planck-Institut für Radioastronomie, Auf dem Hügel 69, 53121 Bonn, Germany

E-mail: eckart@ph1.uni-koeln.de

\section{G.Witzel}

Physics and Astronomy Department, University of California, Los Angeles, CA 90095-1547, USA

\section{N. Sabha}

I.Physikalisches Institut, Universität zu Köln, Zülpicher Str.77, 50937 Köln, Germany

Max-Planck-Institut für Radioastronomie, Auf dem Hügel 69, 53121 Bonn, Germany

\section{Zamaninasab}

Max-Planck-Institut für Radioastronomie, Auf dem Hügel 69, 53121 Bonn, Germany

\begin{abstract}
We report on recent near-infrared observations of the super massive black hole at the Galactic Center (GC), Sagittarius A*. The observations have been carried out using the NACO adaptive optics instrument at the European Southern Observatory's Very Large Telescope on 17th May 2012. The polarization measurements of Sagittarius A* for these data sets show that its emission is polarized during its frequent radiation outbursts. The high level of linear polarization at 2.2 micron and its strong variations can provide clues to constrain the physical conditions of the accretion flow around this SMBH. We are going to combine these data sets with the data simultaneously taken by other telescopes from the $\mathrm{X}$-ray to radio regime. This multiwavelength study will help us reveal the origin of the emission detected from Sagittarius A*.
\end{abstract}

Nuclei of Seyfert galaxies and QSOs - Central engine \& conditions of star formation, November 6-8, 2012

Max-Planck-Insitut für Radioastronomie (MPIfR), Bonn, Germany

\footnotetext{
*Speaker.
} 


\section{Introduction}

The closest super massive black hole (SMBH) lies at the center of the Milky Way with the mass of $4 \times 10^{6} M \odot$, which has been confirmed to exist through the orbital motions of stars around it (Eckart \& Genzel 1996, Genzel et al. 2000, Ghez et al. 1998, Eckart et al. 2002, Schödel et al. 2002, Gillessen et al. 2009). The radio source associated with this SMBH, SagittariusA* (SgrA*), is the best example of low-luminosity AGN accessible to observations. SgrA* is a source of radio, near-infrared (NIR) and X-ray emission (Baganoff et al. 2001, Eckart et al. 2004, Genzel et al. 2003 and Yusef-Zadeh et al. 2006). This source is very faint visible in NIR regime only during its flaring state. Some NIR flares are polarized and have X-ray counterparts which show the radiation mechanism is synchrotron or synchrotron self compton (SSC). Broderick \& Loeb (2006) and Eckart et al. (2006b) modeled the polarized infrared radiation outbursts assuming orbiting hot spots in the inner radii of the accretion flow of the SMBH. Attempts for explaining the radiation from the region around SgrA* have resulted in two different models: jet/jet-base model and hot-spot/disk model. Moreover, multi-wavelength observations of SgrA* can lead us to explain a connection between the NIR flares and the radio emission via adiabatic expansion of source components (Yusef-Zadeh et al. 2006). Here we report on NIR observations using ESO VLT on 17th May 2012. We investigate the polarimetric data taken on this date. We detect variable emission of SgrA* in the 2.2 micron NIR. In Section 2 we describe the observation and data reduction steps. In the following Section we show the photometry results and conclude with a discussion in Section 4.

\section{Observations and data reduction}

The observations of Sgr A* were carried out on 17th May 2012 in the NIR Ks-band (2.0-2.36 $\mu$ m) using the NIR camera CONICA and the adaptive optics (AO) module NAOS on the ESO VLT UT4 on Paranal, Chile. The infrared wavefront sensor of NAOS was used to lock the AO loop on the NIR bright supergiant IRS 7 (Ks-band magnitude $\sim 6.5$ ), located about 5.6 arcsecond north of Sgr A*. In NACOS/CONICA (NACO) the combination of a Wollaston prism with a half-wave retarder plate allows the measurement of two orthogonal directions of the electric field vector. On 17th May 2012, we had a good seeing and the result was diffraction limited images. As a first step, for all the exposures we did sky subtraction, flatfielding and correction for dead or bad pixels (Figure 1). Then we extracted the point spread functions (PSFs) from all the single images by using IDL Star Finder code (Diolaiti et al. 2000). The PSFs were extracted from nearby sources to SgrA*. The individual exposures were deconvolved with the Lucy-Richardson (LR) deconvolution algorithm. We aligned four polarization channels for the polarization data with a cross correlation algorithm . Beam restoration was applied with a Gaussian beam of FWHM corresponding to the final resolution at $2.2 \mu \mathrm{m}$ of 60 milliarcseconds (Figure 2). The flux densities of compact sources were measured via aperture photometry ( 13 constant calibrators and 8 background apertures, Witzel et al. 2012).

\section{Photometric results}

After performing aperture photometry, we obtained the flux densities of calibrators located near SgrA* and also at the position of SgrA*. Figure 3 shows the result of the reduction process, 


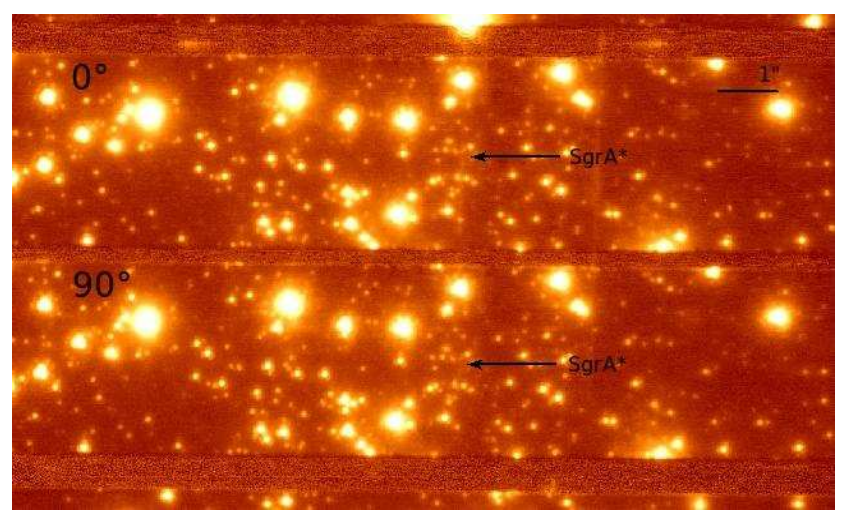

Figure 1: Reduced image of polarimetric observations of GC in NIR Ks-band with the Wollaston prism of NACO on 17th May 2012. The upper stripe shows the ordinary beam of Wollaston prism.

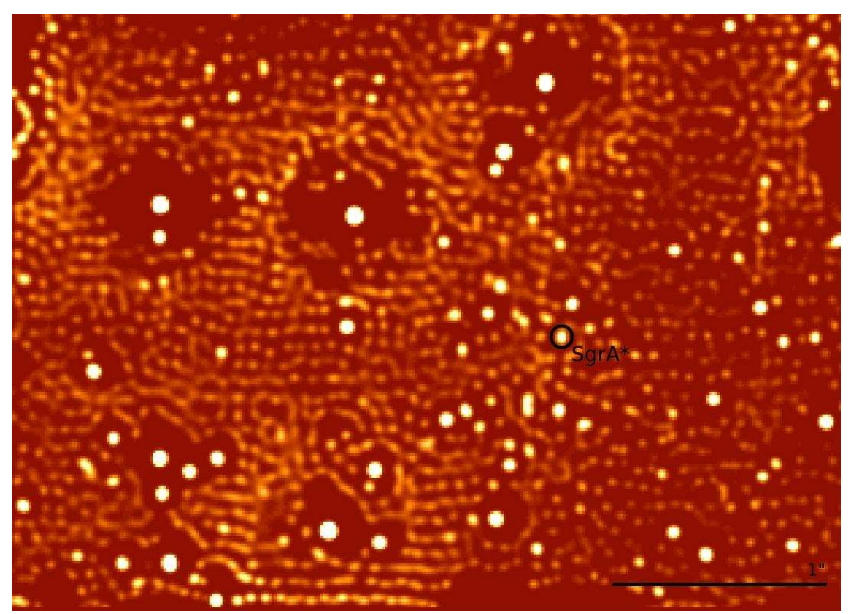

Figure 2: Deconvolved image of the GC on 17th May 2012. The position of SgrA* is indicated by a circle.

the light curve of SgrA* in two orthogonal polarization channels on 17th May 2012. The gaps in the light curves are due to the sky measurements. The flare activity is happening during the first 50 minutes.

\section{Discussion}

The correlations between intensity and polarimetric parameters of the observed light curve can lead us to understand the physical process involved in the emission of SgrA*. Therefore, we obtained the polarimetric parameters for 2012 data. The result is presented in Figure 4, where we can see the polarized flux, polarization degree and polarization angle of SgrA*. The mean polarization degree is about $20 \%$. The flare activity is strong enough to allow studying the polarization measurments in more details and doing the data analysis to better investigate the emission from the vicinity of the black hole. Modeling the NIR polarized data relativisticly (Zamaninasab et al. 2010) and also the spectral energy distribution SED modeling of multi-wavelength data (combining this data with the data taken simultanously by other telescopes in other frequencies) can lead us to understand the exact nature of the origin of the radiation process. 

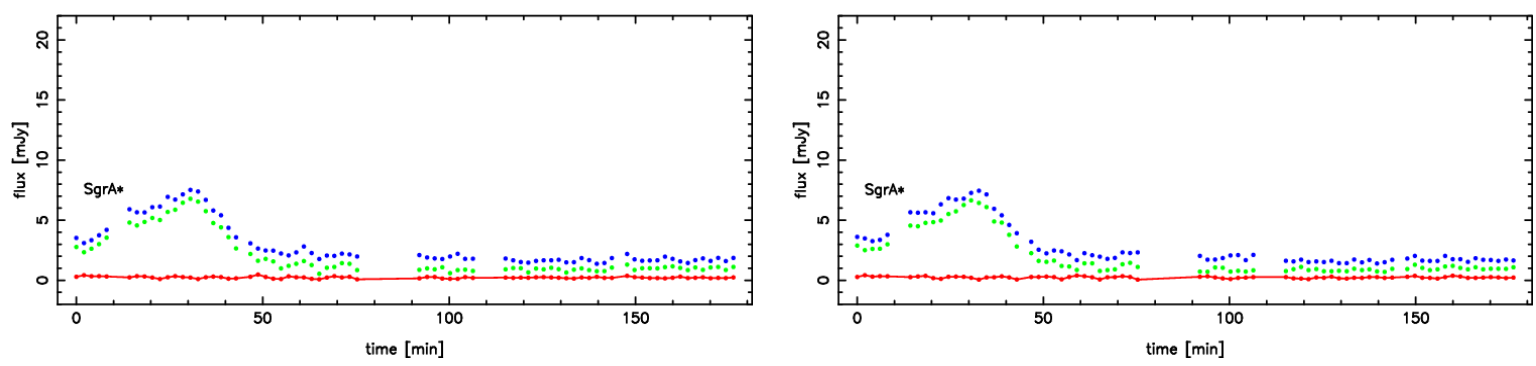

Figure 3: The light curve of SgrA* observed in NIR Ks-band $(2.2 \mu \mathrm{m}$ ) polarimetry mode (orthogonal polarization channels; left: $00^{\circ}, 90^{\circ}$ and right: $45^{\circ}, 135^{\circ}$ ) on 17 th May 2012. The green color shows SgrA* flux density measured in mJy; for a better representation we added the flux of a constant reference star (S17) to SgrA* flux density (blue). Red dots show the background flux densities.

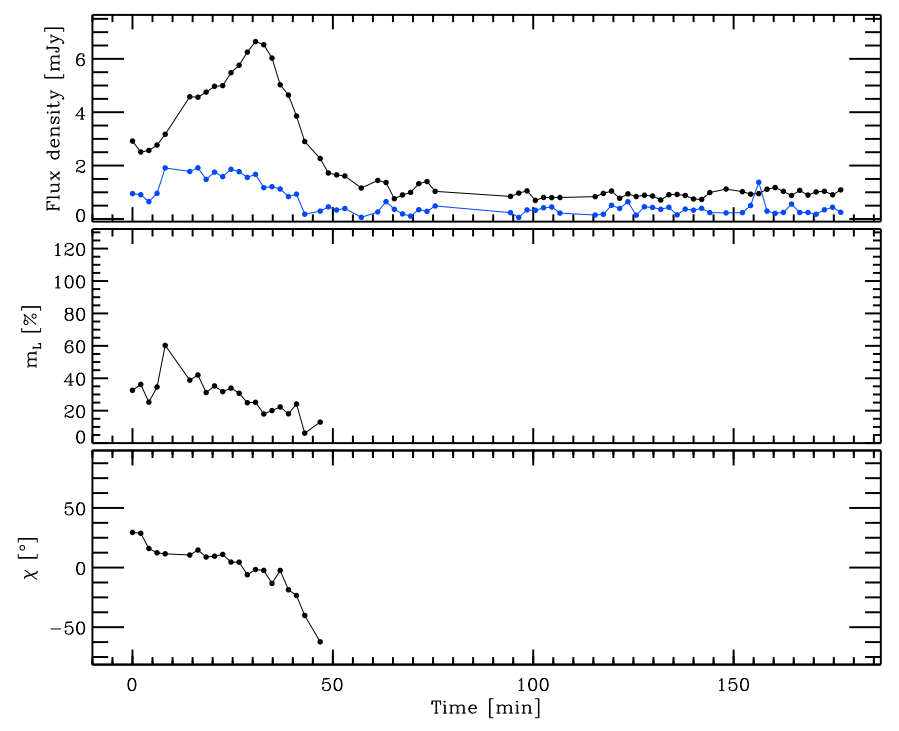

Figure 4: In the top panel black dotes show the flare event of SgrA* and blue dotes show the polarized flux density of this source; Middle panel shows the linear polarization degree and the bottom shows the polarization angle on 17th May 2012. For polarization degree and angle the first 50 minutes is well defined while after 50 minutes is not well defined since the flux variability is low.

\section{References}

[1] Baganoff, F. K., Bautz, M. W., Brandt, W. N., et al., Rapid X-ray flaring from the direction of the supermassive black hole at the Galactic Centre, 2001, Nature, 413, 45.

[2] Broderick, A. E., and Loeb, A., Imaging optically-thin hotspots near the black hole horizon of Sgr A* at radio and near-infrared wavelengths, 2006a, MNRAS, 367, 905

[3] Diolaiti, E., Bendinelli, O., Bonaccini, D., et al., StarFinder: an IDL GUI-based code to analyze crowded fields with isoplanatic correcting PSF fitting, 2000, in Presented at the Society of Photo-Optical Instrumentation Engineers (SPIE) Conference, Vol. 4007, Proc. SPIE Vol. 4007, p. 879-888, Adaptive Optical Systems Technology, Peter L. Wizinowich; Ed., ed. P. L. Wizinowich, 879-888 
[4] Eckart, A., Genzel, R., Observations of stellar proper motions near the Galactic Centre, 1996, Nature, 383, 415

[5] Eckart, A., Genzel, R., Ott, T. and Schödel, R., Stellar orbits near Sagittarius A*, 2002, MNRAS, 331,917

[6] Eckart, A., Baganoff, F. K., Morris, M., et al.,First simultaneous NIR/X-ray detection of a flare from $\operatorname{Sgr} A^{*}, 2004$, A\&A, 427, 1

[7] Eckart, A., Schödel, R., Meyer, L., Trippe, S., et al., Polarimetry of near-infrared flares from Sagittarius A*, 2006b, A\&A, 455, 1

[8] Gillessen, S., Eisenhauer, F., Trippe, S., et al., Monitoring Stellar Orbits Around the Massive Black Hole in the Galactic Center, 2009, ApJ, 692, 1075

[9] Genzel, R., Pichon, C., Eckart, A., et 1., Stellar dynamics in the Galactic Centre: proper motions and anisotropy, 2000, MNRAS, 317, 348

[10] Genzel, R., Schödel, R., Ott, T., et al., Near-infrared flares from accreting gas around the supermassive black hole at the Galactic Centre, 2003b, Nature, 425, 934

[11] Ghez, A., Klein B L, Morris M., and Becklin, E. E., High Proper-Motion Stars in the Vicinity of Sagittarius A*: Evidence for a Supermassive Black Hole at the Center of Our Galaxy, 1998, ApJ, 509,678

[12] Schödel, R., Ott, T., Genzel, R., et al., A star in a 15.2-year orbit around the supermassive black hole at the centre of the Milky Way, 2002, Nature, 419, 694

[13] Wizel, G., Eckart, A., Bremer, M., et al., Source-intrinsic Near infrared properties of SgrA*: Total intensity measurements, 2012, ApJS, 203, 18

[14] Yusef-Zadeh, F., Roberts, D., Wardle, M., et al., Flaring Activity of Sagittarius A* at 43 and 22 GHz: Evidence for Expanding Hot Plasma, 2006, ApJ, 650, 189

[15] Zamaninasab, M., Eckart, A., Witzel, G., et al., Near infrared flares of Sagittarius A*. Importance of near infrared polarimetry, 2010, A\&A, 510, A3 